\title{
Dicionários disponíveis on-line para aprendizes de inglês: estruturação e recursos
}

\author{
Isabel Cristina Tedesco Selistre \\ Doutoranda do Programa de Pós-Graduação em Letras pela \\ Universidade Federal do Rio Grande do Sul - Porto Alegre, \\ RS - Brasil \\ E-mail: ictselistre@yahoo.com
}

\begin{abstract}
Resumo
O artigo descreve a estrutura e as formas de acesso características dos dicionários eletrônicos do tipo on-line disponíveis ao aprendiz de língua inglesa, comparando o conteúdo e os recursos de dois dicionários monolíngues e dois dicionários bilíngües - o Cambridge Advanced Learner's Dictionary (CALD, 2009), o Merriam-Webster Learner's Dictionary (MWLD, 2009), o Reverso Dictionary English/Portuguese (RDEP, 2008) e o Wiktionary Inglês/ Português (WIP, 2009). Discute-se a funcionalidade desses instrumentos em relação às demandas do aprendiz.
\end{abstract}

\section{Palavras-chave}

Dicionários on-line. Aprendizes de inglês. Recursos eletrônicos.

\section{Dictionaries available online for english language learners: structure and resources}

\begin{abstract}
The objective of this paper is to describe the structure and methods of accessing online electronic dictionaries available to students of English Language. The content and resources of two monolingual dictionaries and two bilingual ones have been compared: Cambridge Advanced Learner's Dictionary (CALD, 2009), Merriam-Webster Learner's Dictionary (MWLD, 2009), Reverso Dictionary English/Portuguese (RDEP, 2008) and Wiktionary Inglês/ Português (WIP, 2009)
\end{abstract}

\section{Keywords}

Online dictionaries. English language learners. Electronic resources.

Ci. Inf., Brasília, DF, v. 39 n. 3, p.61-72, set./dez., 2010

\section{INTRODUÇÃO}

Os dicionários eletrônicos, definidos como "qualquer material de referência armazenado em formato eletrônico que forneça informações sobre ortografia, significado ou uso de palavras" (NESI, 2000, p.839), são disponibilizados aos aprendizes de línguas tanto na alternativa off-line quanto na alternativa on-line (dicotomia proposta por Lehr (1996 apud SCHRYVER, 2003)). No primeiro caso, o acesso se dá a partir de dispositivos como dicionários eletrônicos de bolso [pocket electronic dictionaries] e dicionários em CD-ROM; no segundo, o acesso é realizado via interfaces $W e b$, como os dicionários de uso restrito (apenas para membros associados) e os de uso livre apresentados na internet.

Levando em conta que a consulta a dicionários on-line de uso livre é uma prática que, diferentemente da consulta a outros tipos de dicionários eletrônicos, não gera custos, e, principalmente, é uma prática que proporciona ao usuário o acesso rápido a várias fontes similares de pesquisa, possibilitando a comparação e a complementação de informações, propusemo-nos, neste trabalho, a investigar os dicionários disponíveis on-line para os aprendizes de língua inglesa.

Iniciamos descrevendo a composição desses instrumentos (macroestrutura, microestrutura, medioestrutura e material adicional) e as opções de acesso às informações que oferecem e, paralelamente, traçamos um comparativo com os dicionários impressos. $\mathrm{Na}$ sequência, analisamos o conteúdo e os recursos de pesquisa de dois dicionários monolíngues e dois dicionários bilíngues (os quais se encontram identificados na segunda seção do artigo). Por fim, com base em resultados de pesquisas sobre o uso de dicionários on-line, discorremos sobre a funcionalidade dos recursos dessas ferramentas. 
1DICIONÁRIOS ON-LINE: CLASSIFICAÇÃO E COMPOSIÇÃO

\subsection{Classificação}

Os dicionários on-line podem ser classificados como dicionários prontos ou dicionários em construção (STORRER; FREESE, 1996 apud WELKER, 2004, p.228). Os dicionários prontos correspondem às versões eletrônicas de dicionários impressos e aos dicionários especialmente concebidos para aproveitar as vantagens do hipertexto. Os dicionários em construção compreendem os dicionários que fornecem a parte do trabalho já concluída pelo(s) seu(s) autor(es) e os dicionários colaborativos, que são desenvolvidos a partir de contribuições dos seus usuários.

Os dicionários prontos e os dicionários em construção com autoria reconhecida pelos seus pares são obras que apresentam alto grau de confiabilidade, uma vez que são elaborados por especialistas qualificados (lexicógrafos, linguistas, pedagogos e outros) e, comumente, abonados por editoras respeitadas no mercado. Já os dicionários colaborativos, embora possam trazer informações úteis ao consulente, não representam fontes lexicográficas fiáveis, pois carecem de supervisão profissional.

Independentemente da classe, todo dicionário on-line, tal como os dicionários impressos, é constituído por uma macroestrutura, uma microestrutura, uma medioestrutura e material complementar.

\subsection{Composição}

\subsubsection{Macroestrutura}

A macroestrutura corresponde à "estrutura global da lista que permite ao redator e ao usuário localizar informações em uma obra de referência" (HARTMANN; JAMES, 2001). Em outras palavras, a macroestrutura corresponde ao conjunto de itens lexicais (referidos nos estudos lexicográficos como 'lemas' ou 'entradas') arrolados em um dicionário.
Os mesmos tipos de itens lexicais arrolados em um dicionário impresso são passíveis de inclusão na macroestrutura de um dicionário on-line:

a) lexias ${ }^{1}$ simples - formas canônicas (formas básicas como house, child, eat e good);

b) lexias simples - formas não-canônicas (formas flexionadas como ate, children e best);

c) lexias compostas (como brother-in-law e fying saucer);

d) lexias complexas (como to freeze to death e kick the bucket);

e) formas truncadas ${ }^{2}$ (como photo e ad);

f) siglas (como FDA e $N A S A)$;

g) abreviaturas (como Ltd e Co.);

h) formas contraídas (como ain't e won't);

i) nomes próprios: topônimos (como Finland) e antropônimos (como Jane Doe);

j) marcas registradas (como Band-Aid e Coke);

k) afixos (como self- e -ful).

Em relação à estrutura de acesso, verifica-se que os dicionários eletrônicos liberam o usuário da busca alfabética linear (LEECH; NESI, 1999) - única rota de pesquisa possível nos dicionários impressos - e oferecem, em geral, uma série de opções de pesquisa:

a) browsing: equivalente eletrônico à pesquisa em dicionário de papel - o usuário percorre uma lista alfabética até encontrar o item de seu interesse (HALL, 2000, p.77);

b) busca direta: o usuário digita um item lexical na caixa de busca e tem acesso direto ao conteúdo da entrada (HALL, 2000, p.78);

\footnotetext{
${ }^{1}$ Pelo fato de o conceito de 'palavra' ser muito abrangente (e, por isso, vago) e o termo 'lexema' ser de domínio da lexicologia, optamos pelo termo 'lexia' (proposto por Pottier em 1974). ${ }^{2}$ Sobre o fenômeno de 'truncação', ver Peruzzo (2007).
}

Ci. Inf., Brasília, DF, v. 39 n. 3, p.61-72, set./dez., 2010 
c) furay search: caso o usuário digite incorretamente um item, o dicionário oferece uma lista de opções cuja grafia se aproxime ao que foi solicitado (RIZORODRÍGUEZ, 2008);

d) wildcards: quando o usuário tiver dúvidas em relação à grafia do item, poderá substituir a letra em questão por '?', ou utilizar '*' para qualquer outro número de caracteres desconhecidos; esse tipo de busca também pode trazer os derivativos do item em questão (JACKSON, 2002, p.70).

\subsubsection{Microestrutura}

A microestrutura é "a parte em que são organizadas todas as informações a serem mencionadas acerca do lema" (CARVALHO, 2001, p.65); é onde, segundo Bugueño e Farias (2006, p. 116), encontramos as informações acerca do item lexical arrolado na macroestrutura tanto na sua condição de significante - 'comentário de forma' - quanto de significado - 'comentário semântico'.

\subsubsection{Componentes da microestrutura}

No comentário de forma dos dicionários para aprendizes, qualquer que seja o suporte, comumente temos indicações de ortografia (grafia convencional do item), de divisão silábica (menos frequentemente), de variantes ortográficas, de pronúncia e informações gramaticais (categoria gramatical e, quando é o caso, apresentação das formas flexionadas não previsíveis).

O comentário semântico traz definições (nos dicionários monolíngues) equivalentes (nos dicionários bilíngues), marcas de uso, desambiguadores semânticos e, em algumas entradas, podem apresentar ilustrações.

Os exemplos, conforme Farias (2008), podem servir para demonstrar o emprego sintático do lema ou para reforçar a compreensão do seu significado. Assim, podemos dizer que, no primeiro caso, o exemplo faz parte do comentário de forma e, no segundo, faz parte do comentário semântico.

\subsubsection{Tipos de microestrutura}

Além do comentário de forma e do comentário semântico, a microestrutura acomoda os fraseologismos (expressões idiomáticas, fórmulas situacionais, frases feitas, provérbios e colocações etc.).

A forma de organizar esses fraseologismos pode diferir de um dicionário para outro. Hausmann; Werner (1991 apud CARVALHO 2001, p.156-160) dividem as microestruturas básicas em três tipos:

a) integrada - as respectivas informações sintagmáticas são apresentadas após cada acepção;

b) não-integrada - as informações sintagmáticas são separadas das diversas acepções, aparecendo no final do verbete, às vezes, num bloco à parte (em alguns casos, esses sintagmas recebem números que se referem à acepção à qual pertencem, permitindo assim melhor identificação);

c) parcialmente integrada - é como a microestrutura integrada, porém alguns sintagmas são colocados no final, num parágrafo ou bloco à parte, porque não está claro à qual acepção pertencem.

\subsubsection{Medioestrutura}

A medioestrutura é o sistema de remissões (ou referências cruzadas) do dicionário, isto é, o sistema que remete o usuário de uma parte do dicionário a outra (BUGUEÑO, 2003, p.108).

As remissões, segundo Wiegand (1996 apudWELKER, 2004, p. 178-179), podem ser obrigatórias, quando o usuário recebe a informação desejada apenas se seguir a remissão (por exemplo, a remissão de uma forma flexionada para a sua forma canônica); ou facultativas, quando o consulente segue a remissão se desejar informações adicionais (como no caso das remissões para informações complementares: resumos gramaticais, ilustrações etc.).

Nos dicionários on-line, dada a possibilidade de hiperconexões (byperlinks), o cruzamento de referências é muito mais abrangente do que nos dicionários impressos. 


\subsubsection{Material complementar}

Como material complementar, os dicionários para aprendizes usualmente apresentam:

a) uma introdução ou prefácio com informações sobre a elaboração do dicionário;

b) um guia para o usuário, que traz o tipo de informações incluídas no dicionário e a forma de serem acessadas;

c) listas de verbos irregulares, nomes de países e nacionalidades, tabelas de pesos e medidas, apêndices gramaticais, minidicionários ilustrados, etc., no meio ou no final da obra.

Nos dicionários on-line, como não há problemas de espaço, o número de informações adicionais pode ser ampliado. Além do material já citado, há possibilidade de incluir jogos interativos, material fotocopiável com atividades extras e acesso a outros dicionários.

Após essa breve revisão sobre a classificação, a composição e os recursos dos dicionários on-line, passemos, agora, à descrição e análise de quatro desses dicionários.

\section{CONTEÚDO E RECURSOS DE QUATRO DICIONÁRIOS ON-LINE}

A seleção dos dicionários para este estudo foi efetuada do seguinte modo:

a) dicionários monolíngues: inicialmente, buscamos no Google os itens online dictionaries e consideramos o primeiro resultado indicado - Cambridge Dictionaries Online (inglês britânico); partimos, então, para uma segunda pesquisa, buscando os itens american online dictionaries e, novamente, consideramos o primeiro resultado - Merriam-Webster Online (inglês americano);

b) para os dicionários bilíngues: decidimos fazer a seleção a partir de um site bastante utilizado por todo tipo de aprendiz ${ }^{3}$ - a Wikipedia; pesquisamos

${ }^{3}$ O site Wikipedia é o $7^{\circ}$. mais acessado da Web, conforme Alexa - The Web Information Company <http://www.alexa.com/topsites > Acesso em: 28 ago. 2010. Porém, se considerarmos a categoria enciclopédia, o verbete electronic dictionaries ${ }^{4}$ e selecionamos dois dicionários citados na lista de referências ${ }^{5}$ - o Wiktionary, que aparece no topo da lista e o Reverso Dictionary, o primeiro dicionário organizado por editora reconhecida - a Collins - citado nas referências.

Nos quadros seguintes, identificamos os editores e a tipologia dos quatro dicionários.

\section{QUADRO 1}

\section{Dicionários monolíngues pesquisados}

\begin{tabular}{l|l|l}
\hline \multicolumn{1}{c|}{ Dicionário } & \multicolumn{1}{c|}{ Editor } & \multicolumn{1}{c}{ Tipo } \\
\hline $\begin{array}{l}\text { Cambridge Advanced Learner's } \\
\text { Dictionary (CALD, 2009) }\end{array}$ & $\begin{array}{l}\text { Cambridge University } \\
\text { Press }\end{array}$ & $\begin{array}{l}\text { versão eletrônica de } \\
\text { dicionário impresso }\end{array}$ \\
\hline $\begin{array}{l}\text { Merriam Webster Learner's } \\
\text { Dictionary (MWLD, 2009) }\end{array}$ & $\begin{array}{l}\text { Merriam-Webster } \\
\text { Incorporated }\end{array}$ & $\begin{array}{l}\text { versão eletrônica de } \\
\text { dicionário impresso }\end{array}$ \\
\hline
\end{tabular}

\section{QUADRO 2}

\section{Dicionários bilíngues pesquisados}

\begin{tabular}{l|l|l}
\hline \multicolumn{1}{c|}{ Dicionário } & \multicolumn{1}{c|}{ Editor } & \multicolumn{1}{c}{ Tipo } \\
\hline $\begin{array}{l}\text { Reverso Dictionary } \\
\text { English/Portuguese } \\
\text { (RDEP, 2008) }\end{array}$ & $\begin{array}{l}\text { Harper Collins } \\
\text { Publishers }\end{array}$ & $\begin{array}{l}\text { versão eletrônica } \\
\text { de dicionário } \\
\text { impresso }\end{array}$ \\
\hline $\begin{array}{l}\text { Wiktionário } \\
\text { Inglês/Português } \\
\text { (WIP, 2009) }\end{array}$ & $\begin{array}{l}\text { contribuições } \\
\text { de usuários }\end{array}$ & $\begin{array}{l}\text { dicionário } \\
\text { colaborativo }\end{array}$ \\
\hline
\end{tabular}

Nas próximas seções, apresentamos o conteúdo da macroestrutura, da microestrutura, da medioestrutura e do material complementar desses dicionários.

\subsection{A macroestrutura dos dicionários analisados}

\subsubsection{Itens arrolados}

Tomando por base a relação dos tipos de itens passíveis de lematização em um dicionário para aprendizes de inglês listada em 1.2.1, verificamos o seguinte:

- os quatro dicionários - CALD (2009), MWLD (2009), RDEP (2008) e WIP (2009) - arrolam:

é o primeiro.

${ }^{4}<$ http://en.wikipedia.org/wiki/Electronic dictionary $>$ Acesso em: 28 ago. 2010.

${ }^{5}<$ http://en.wikipedia.org/wiki/Electronic_dictionary\#References> Acesso em: 28 ago. 2010.

Ci. Inf., Brasília, DF, v. 39 n. 3, p.61-72, set./dez., 2010 
lexias simples na forma canônica (forma básica) e na forma não-canônica (forma flexionada), lexias compostas, lexias complexas, formas truncadas, siglas, abreviaturas, formas contraídas e marcas registradas;

- apenas o CALD não inclui topônimos na sua macroestrutura;

- somente o RDEP não lista antropônimos;

- não aparecem afixos no RDEP nem no WIP, embora nesse último haja um campo designado para esta categoria aguardando contribuições.

\section{QUADRO 3}

Macroestrutura: tipo de item arrolado

\begin{tabular}{l|c|c|c|c}
\hline & $\begin{array}{c}\text { CALD } \\
(2009)\end{array}$ & $\begin{array}{c}\text { MWLD } \\
(2009)\end{array}$ & $\begin{array}{c}\text { RDEP } \\
(2008)\end{array}$ & $\begin{array}{c}\text { WIP } \\
(2009)\end{array}$ \\
\hline $\begin{array}{l}\text { lexias simples - } \\
\text { formas canônicas: }\end{array}$ & $\sqrt{ }$ & $\sqrt{ }$ & $\sqrt{ }$ & $\sqrt{ }$ \\
\hline $\begin{array}{l}\text { lexias simples - } \\
\text { formas não-canônicas }\end{array}$ & $\sqrt{ }$ & $\sqrt{ }$ & $\sqrt{ }$ & $\sqrt{ }$ \\
\hline lexias composta & $\sqrt{ }$ & $\sqrt{ }$ & $\sqrt{ }$ & $\sqrt{ }$ \\
\hline lexias complexas & $\sqrt{ }$ & $\sqrt{ }$ & $\sqrt{ }$ & $\sqrt{ }$ \\
\hline formas truncadas & $\sqrt{ }$ & $\sqrt{ }$ & $\sqrt{ }$ & $\sqrt{ }$ \\
\hline siglas & $\sqrt{ }$ & $\sqrt{ }$ & $\sqrt{ }$ & $\sqrt{ }$ \\
\hline abreviaturas & $\sqrt{ }$ & $\sqrt{ }$ & $\sqrt{ }$ & $\sqrt{ }$ \\
\hline formas contraídas & $\sqrt{ }$ & $\sqrt{ }$ & $\sqrt{ }$ & $\sqrt{ }$ \\
\hline nomes: topônimos & $\varnothing$ & $\sqrt{ }$ & $\sqrt{ }$ & $\sqrt{ }$ \\
\hline nomes: antropônimos & $\sqrt{ }$ & $\sqrt{ }$ & $\varnothing$ & $\sqrt{ }$ \\
\hline marcas registradas & $\sqrt{ }$ & $\sqrt{ }$ & $\sqrt{ }$ & $\sqrt{ }$ \\
\hline afixos & $\sqrt{ }$ & $\sqrt{ }$ & $\varnothing$ & $\varnothing$ \\
\hline
\end{tabular}

\subsubsection{Opções de busca}

a) browsing: o CALD e o RDEP não disponibilizam a busca browsing; o MWLD oferece no canto inferior direito a opção Browse the Learner's Dictionary; o WIP organiza sua macroestrutura em categorias e subcategorias que podem ser consultadas através da pesquisa alfabética;

b) busca direta: os quatro dicionários permitem a busca direta;

c) fur:y search: para testar a busca fur:y, digitamos na caixa de busca os itens insight, thesaurus e brother sem o ' $h$ '; os resultados diferiram bastante de um dicionário para outro: o CALD listou 87 opções para o primeiro item (insight aparece na $70^{\circ}$ posição), 22 para o segundo (thesaurus em $10^{\circ}$ ) e 42 para o terceiro item (brother aparece em $30^{\circ}$ ); o MWLD apresentou 13 opções para o primeiro caso (insight é a $1^{\mathrm{a}}$. opção), 5 para o segundo e 20 para o terceiro, porém, nenhuma das opções estava correta para thesaurus nem para brother, o RDEP disponibilizou 5 opções para cada um dos itens, entretanto, a busca foi bem-sucedida apenas para brother, o WIP funcionou perfeitamente para os dois primeiros itens, apresentando apenas uma opção, mas não trouxe nenhum resultado coerente para brother,

d) wildcards: utilizamos help* e *ful para verificar a busca wildcards. O CALD disponibilizou apenas as entradas help e -ful; o MWLD, listou 10 itens para cada caso (help, help desk, help desks, helped, helper, belpers, belpful, belpfully, helpfulness, helpfulnesses e allpowverful, armful, armsful, artful, awful, bagful, baleful, baneful, bashful, basketful); o RDEP apresentou 5 possibilidades para belp* (help, helper, belpful, belping, helpless), mas nenhuma opção coerente para *ful; o WIP, apesar de não apresentar nada para help*, foi bastante produtivo para *ful (deceitful, powerful, sucessful, careful, beautiful, etc.).

Esses testes demonstraram que tanto a busca furzy como a busca wildcards nem sempre são bem-sucedidas. Isso não invalida o recurso, uma vez que em boa parte dos casos obtivemos bons resultados.

QUADRO 4

Macroestrutura: facilidades de busca

\begin{tabular}{l|c|c|c|c}
\hline & $\begin{array}{c}\text { CALD } \\
(2009)\end{array}$ & $\begin{array}{c}\text { MWLD } \\
(\mathbf{2 0 0 9 )}\end{array}$ & $\begin{array}{c}\text { RDEP } \\
\mathbf{( 2 0 0 8 )}\end{array}$ & $\begin{array}{c}\text { WIP } \\
\mathbf{( 2 0 0 9 )}\end{array}$ \\
\hline browsing & $\varnothing$ & $\sqrt{ }$ & $\varnothing$ & $\sqrt{ }$ \\
\hline busca direta & $\sqrt{ }$ & $\sqrt{ }$ & $\sqrt{ }$ & $\sqrt{ }$ \\
\hline furisy search & $\sqrt{ }$ & $\sqrt{ }$ & $\sqrt{ }$ & $\sqrt{ }$ \\
\hline mildcards & $\varnothing$ & $\sqrt{ }$ & $\sqrt{ }$ & $\sqrt{ }$ \\
\hline
\end{tabular}




\subsection{A microestrutura dos dicionários analisados}

\subsubsection{Elementos microestruturais}

Analisando os elementos microestruturais apresentados nos dicionários, constatamos que:

- os elementos microestruturais comuns aos quatro dicionários são ortografia, categoria gramatical, formas irregulares dos graus dos adjetivos, formas irregulares de passado e particípio passado, marcas de uso e exemplos;

- a divisão silábica é utilizada por dois dos quatro dicionários: o CALD e o RDEP;

- as variantes ortográficas e as formas irregulares de plural não são apresentadas no WIP, e a pronúncia não aparece no RDEP;

- o MWLD e o WIP oferecem ilustrações como recursos microestruturais adicionais em algumas de suas entradas: o WIP disponibiliza as ilustrações na própria entrada; já o MWLD remete o usuário para o minidicionário ilustrado que faz parte do seu material complementar (por exemplo, na entrada avocado encontramos um link no final do verbete para a página do minidicionário que contém ilustrações de frutas).

\section{QUADRO 5}

\section{Microestrutura: componentes}

\subsubsection{Conteúdo microestrutural}

Para avaliar a apresentação dos verbetes e as informações contidas na microestrutura, verificamos a entrada PILE nos quatro dicionários:

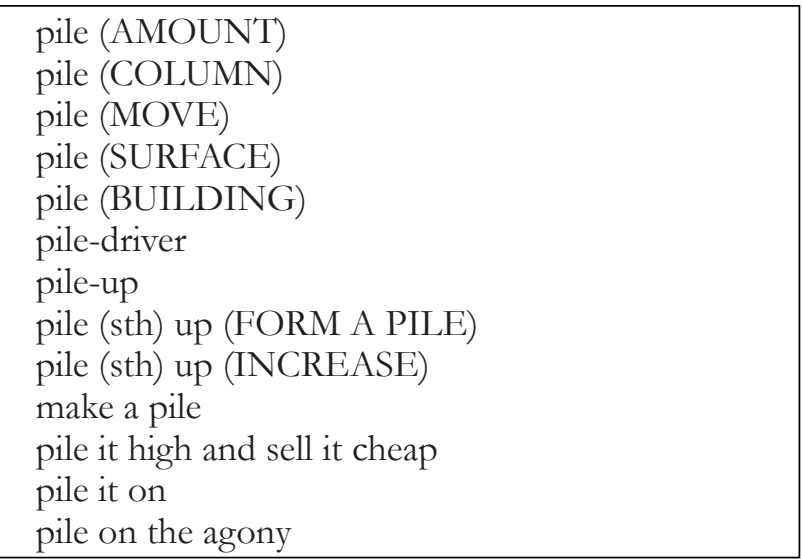

(CALD, 2009, s.v. pile)

pile (COLUMN) Show phonetics noun $[\mathrm{C}]$

a strong column or post of wood, steel or concrete which is pushed into the ground to help support a building

(CALD, 2009, s.v. pile/COLUMN)

${ }^{1}$ pile (noun)

${ }^{2}$ pile (verb)

${ }^{3}$ pile (noun)

${ }^{4}$ pile (noun)

${ }^{1}$ bottom (noun)

pile driver

${ }^{1}$ pound (noun)

${ }^{1}$ top (noun)

\begin{tabular}{|c|c|c|c|c|}
\hline & CALD (2009) & MWLD (2009) & RDEP (2008) & WIP (2009) \\
\hline Ortografia (grafia convencional do item) & $\sqrt{ }$ & $\sqrt{ }$ & $\sqrt{ }$ & $\sqrt{ }$ \\
\hline divisão silábica & $\varnothing$ & $\sqrt{ }$ & $\varnothing$ & $\sqrt{ }$ \\
\hline variantes ortográficas & $\sqrt{ }$ & $\sqrt{ }$ & $\sqrt{ }$ & $\varnothing$ \\
\hline pronúncia & $\sqrt{ }$ & $\sqrt{ }$ & $\varnothing$ & $\sqrt{ }$ \\
\hline categoria gramatical & $\sqrt{ }$ & $\sqrt{ }$ & $\sqrt{ }$ & $\sqrt{ }$ \\
\hline formas irregulares: comparativos e superlativos, & $\sqrt{ }$ & $\sqrt{ }$ & $\sqrt{ }$ & $\sqrt{ }$ \\
\hline formas irregulares : plural & $\sqrt{ }$ & $\sqrt{ }$ & $\sqrt{ }$ & $\varnothing$ \\
\hline formas verbais irregulares & $\sqrt{ }$ & $\sqrt{ }$ & $\sqrt{ }$ & $\sqrt{ }$ \\
\hline Definições (dicionários monolíngues) & $\sqrt{ }$ & $\sqrt{ }$ & - & - \\
\hline equivalentes (dicionários bilíngues) & - & - & $\sqrt{ }$ & $\sqrt{ }$ \\
\hline ilustrações & $\varnothing$ & $\sqrt{ }$ & $\varnothing$ & $\sqrt{ }$ \\
\hline marcas de uso & $\sqrt{ }$ & $\sqrt{ }$ & $\sqrt{ }$ & $\sqrt{ }$ \\
\hline exemplos & $\sqrt{ }$ & $\sqrt{ }$ & $\sqrt{ }$ & $\sqrt{ }$ \\
\hline
\end{tabular}




\section{Pronounced: /'pajəl/ $\mathrm{b}$ )}

Function: noun

Inflected forms: plural piles

Meaning:

[count $1:$ a group of things that are put one on top of another - He put the magazines into a neat pile. - She raked the leaves into piles. - Take a card from the pile. - - often + of $\boldsymbol{\bullet}$ a pile of wood - a pile of clothes

2 informal a : a very large amount of something - She had piles of work to do. "He makes a pile of money. $\mathbf{b}$ : a large amount of money $\cdot \mathrm{He}$ made his pile and then retired. - He made a pile in the stock market.

at the bottom of the pile

: in a low or very unimportant position -

He is at the bottom of the pile but should get a promotion soon. - The team finished the season at the bottom of the pile.

at the top of the pile

: in a high or very important position - With this promotion, he will be at the top of the pile. The team finished the season at the top of the pile. - compare ${ }^{3} \underline{\text { PILE}},{ }^{4} \underline{\text { PILE }}$

(MWLD, 2009, s.v. 1pile)

\begin{tabular}{|l|}
\hline pile \\
$\mathbf{n}$ (of books) pilha, (heap) monte $m$, (of \\
carpet) pêlo, (of cloth) lado \\
felpudo, (support, in building) estaca \\
$\mathbf{v t ~ - ~ p i l e ~ u p ~ e m p i l h a r , ~ ( h e a p ) ~ a m o n t o a r , ~}$ \\
(fig) acumular \\
vi - pile up (objects) empilhar- \\
se, (problems, work) acumular-se \\
in a pile numa pilha \\
$\mathbf{\Delta}$ pile into \\
$\mathbf{v t}$ fus (car) apinhar-se \\
$\mathbf{\Delta}$ pile on \\
$\mathbf{v t}$ \\
to pile it on inf exagerar \\
pile-up \\
$\mathbf{n}$ (aut) engavetamento
\end{tabular}

(RDEP, 2008, s.v. pile)

pile

pilha

amontoado

Categoria: Substantivo (Inglês)

(WIP, 2009, s.v. pile)
No CALD e no MWLD, o consulente faz a pesquisa do verbete em duas etapas: na primeira, após digitar um item na caixa de busca, obtém, num painel longo e estreito à esquerda, uma lista de opções que contêm o item digitado; na segunda, depois de clicar na opção que lhe interessa, acessa as informações microestruturais sobre ela.

Embora os dois dicionários tenham o mesmo esquema de apresentação, o CALD é bem elaborado do que o MWLD. No primeiro, cada opção listada vem acompanhada de um desambiguador e as fraseologias são listadas à parte, no final da janela, arranjo que adiciona simplicidade às entradas e facilita a personalização, ou seja, a adaptação aos objetivos da pesquisa de cada usuário. No segundo, as opções são acompanhadas apenas de distinções gramaticais; o usuário tem de abrir cada uma delas até chegar ao conteúdo referente às suas necessidades.

O RDEP e o WIP exibem, assim como os dicionários impressos, todo o conteúdo microestrutural de uma vez, fazendo com que o usuário tenha de percorrer todo texto até encontrar o que está buscando.

O layout das entradas nos quatro dicionários é, evidentemente, superior ao das obras impressas, pois os dicionários eletrônicos não têm de lidar com a questão do espaço. A palavra entrada claramente evidenciada, a utilização de recuo para significados distintos, exemplos e fraseologias, o espaçamento generoso e a utilização de cores e/ou fontes diferentes contribuem para a clareza e nitidez do texto.

Examinando o conteúdo microestrutural dos verbetes selecionados, percebemos que: (1) as informações fornecidas no comentário de forma são basicamente as mesmas nos dicionários monolíngues e nos bilíngues; (2) no comentário semântico dos dicionários monolíngues, como era de se esperar (uma vez que têm como usuário-alvo aprendizes de nível mais avançado), encontramos informações muito mais complexas do que nos dicionários bilíngues. 
A comparação que realizamos entre os dois dicionários bilíngues evidenciou as deficiências de um dicionário do tipo colaborativo; observamos que o WIP não apresenta marcas de uso, nem phrasal verbs ou fraseologias, o que torna sua consulta bastante restrita.

\subsubsection{Tipos de microestrutura}

O tipo de microestrutura é o mesmo nos quatro dicionários: as fraseologias figuram no final do verbete. Tal arranjo, acreditamos, facilita a pesquisa do usuário.

\section{QUADRO 6}

Tipos de microestrutura

\begin{tabular}{|l|c|c|c|c|}
\hline & $\begin{array}{l}\text { CALD } \\
(2009)\end{array}$ & $\begin{array}{l}\text { MWLD } \\
(2009)\end{array}$ & $\begin{array}{l}\text { RDEP } \\
(2008)\end{array}$ & $\begin{array}{l}\text { WIP } \\
(2009)\end{array}$ \\
\hline Integrada & $\varnothing$ & $\varnothing$ & $\varnothing$ & $\varnothing$ \\
\hline Não integrada & $\sqrt{ }$ & $\sqrt{ }$ & $\sqrt{ }$ & $\sqrt{ }$ \\
\hline $\begin{array}{l}\text { Parcialmente } \\
\text { integrada }\end{array}$ & $\varnothing$ & $\varnothing$ & $\varnothing$ & $\varnothing$ \\
\hline
\end{tabular}

\subsection{A medioestrutura dos dicionários analisados}

Neste estudo identificamos oito tipos de remissões (as mesmas que encontramos nos dicionários impressos): da forma verbal não-canônica para a canônica, da forma irregular de adjetivo comparativo e superlativo para a forma canônica, da forma irregular de plural para a forma canônica, da forma contraída para a forma canônica, das formas truncadas para forma extensa, de uma forma variante à outra, de um item base para seus compostos e remissões para o material complementar.

As definições (nos dicionários monolíngues) e os equivalentes (nos dicionários bilíngues) aparecem tanto na forma canônica quanto na forma nãocanônica dos adjetivos, mas apenas nas formas canônicas dos verbos e dos substantivos.

O MWLD e o WIP são os dois dicionários que trazem ilustrações; todavia, somente o primeiro utiliza remissão (por exemplo: stapler remete para a página office, que traz ilustrações de objetos relacionados ao tópico escritório).

Ao final de cada definição de um item base, o MWLD oferece links para os seus compostos (por exemplo, em office remete para box office, oval office, ticket office, etc.).

O CALD, o MWLD e o RDEP remetem das formas contraídas e das formas truncadas para as formas extensas, onde o consulente encontra o significado do item; o WIP não faz este tipo de remissão.

E, por fim, as variantes, que não são apresentadas apenas no dicionário colaborativo, informam a definição ou equivalência em apenas uma das entradas.

\section{QUADRO 7}

\section{Medioestrutura: tipo de remissões}

\begin{tabular}{|l|c|c|c|c|}
\hline & $\begin{array}{c}\text { CALD } \\
\text { (2009) }\end{array}$ & $\begin{array}{l}\text { MWLD } \\
\text { (2009) }\end{array}$ & $\begin{array}{c}\text { RDEP } \\
\text { (2008) }\end{array}$ & $\begin{array}{l}\text { WIP } \\
\text { (2009) }\end{array}$ \\
\hline $\begin{array}{l}\text { Forma verbal } \\
\text { não-canônica } \\
\text { para a canônica }\end{array}$ & $\sqrt{ }$ & $\sqrt{ }$ & $\sqrt{ }$ & $\sqrt{ }$ \\
\hline $\begin{array}{l}\text { Forma irregular } \\
\text { adjetivo } \\
\text { para a forma } \\
\text { canônica }\end{array}$ & $\sqrt{ }$ & $\sqrt{ }$ & $\sqrt{ }$ & $\sqrt{ }$ \\
\hline $\begin{array}{l}\text { Forma irregular } \\
\text { de plural } \\
\text { p/ a forma } \\
\text { canônica }\end{array}$ & $\sqrt{ }$ & $\sqrt{ }$ & $\sqrt{ }$ & $\sqrt{ }$ \\
\hline $\begin{array}{l}\text { Forma contraída } \\
\text { p/ forma } \\
\text { canônica }\end{array}$ & $\sqrt{ }$ & $\sqrt{ }$ & $\varnothing$ \\
\hline $\begin{array}{l}\text { Formas } \\
\text { truncadas } \\
\text { p/ forma } \\
\text { extensa }\end{array}$ & $\sqrt{ }$ & $\sqrt{ }$ & $\sqrt{ }$ & $\emptyset$ \\
\hline $\begin{array}{l}\text { De uma variante } \\
\text { à outra }\end{array}$ & $\sqrt{ }$ & $\sqrt{ }$ & $\sqrt{ }$ & - \\
\hline $\begin{array}{l}\text { Itens base para } \\
\text { seus compostos }\end{array}$ & $\varnothing$ & $\sqrt{ }$ & $\varnothing$ & $\varnothing$ \\
\hline $\begin{array}{l}\text { Material } \\
\text { complementar }\end{array}$ & - & $\sqrt{ }$ & - & $\varnothing$ \\
\hline
\end{tabular}

Ci. Inf., Brasília, DF, v. 39 n. 3, p.61-72, set./dez., 2010 


\subsection{O material complementar dos dicionários analisados}

Quanto ao material complementar:

- o CALD traz informações sobre a elaboração do dicionário na guia find out more; o RDEP e o WIP, na página inicial; o MWLD não disponibiliza nenhum dado sobre este tópico;

- os quatro dicionários oferecem orientações para o usuário: o CALD na guia help, o MWLD na guia Using the Learner's Dictionary, o RDEP na página inicial e o WIP na guia 'Ajuda';

- somente o RDEP não inclui apêndices de vocabulário e/ou gramática: no CALD temos as Top 40 words (lista com as 40 entradas mais clicadas no último mês) e a Word of the day (a cada dia um item novo é destacado juntamente com suas definições e exemplos de uso); no MWLD encontramos as 3,000 words (as palavras mais frequentes da língua inglesa), a Learner's Word of the Day (semelhante ao CALD) e a Language Help (que arrola os Common Word Usage Problems e as Words Often Confused, além de um Grammar Glossary); no WIP há um rol de 'Palavras inglesas com sinais diacríticos'(fiancé, fiancée etc.) e uma 'Gramática Inglesa' que traz 'Preposição - Lista Português-Inglês', 'Sufixos da Língua Inglesa', 'Verbos: conjugação - e 'Verbos irregulares da Língua Inglesa';

- o MWLD é o único que disponibiliza atividades on-line (Perfect Pronunciation Exercises);

- os dois dicionários monolíngues sugerem várias atividades (com chave de resposta) que podem ser reproduzidas;

- apenas o CALD incorpora material para o professor: 10 planos de aula;

- o CALD possibilita que o usuário pesquise em mais cinco dicionários: Cambridge Learner's Dicionary, Cambridge Dictionary of American English, Cambridge International Dictionary of Idioms, Cambridge International Dictionary of Phrasal Verbs e Visual Thesaurus; o
MWLD agrega o Visual Dictionary, a Encyclopedia Brittanica, o Merriam-Webster Online e o MerriamWebster's Word Central (for kids); o RDEP permite o acesso a quatro dicionários monolíngues: English Definitions, English Conjugation, English Synonyms e English for learners, além de um Collaborative Dictionary.(bilíngue); o WIP inclui um Dicionário Fraseológico Inglês/Português - Português/ Inglês, um Dicionário Ilustrado (semelhante a uma enciclopédia) e um Thesaurus.

\section{QUADRO 8 \\ Material complementar: conteúdo}

\begin{tabular}{|l|c|c|c|c|}
\hline & $\begin{array}{c}\text { CALD } \\
(2009)\end{array}$ & $\begin{array}{c}\text { MWLD } \\
(\mathbf{2 0 0 9 )}\end{array}$ & $\begin{array}{c}\text { RDEP } \\
(\mathbf{2 0 0 1 8}\end{array}$ & $\begin{array}{c}\text { WIP } \\
(2009)\end{array}$ \\
\hline $\begin{array}{l}\text { Informações s/ } \\
\text { elaboração do } \\
\text { dicionário }\end{array}$ & $\sqrt{ }$ & $\varnothing$ & $\sqrt{ }$ & $\sqrt{ }$ \\
\hline Guia do usuário & $\sqrt{ }$ & $\sqrt{ }$ & $\sqrt{ }$ & $\sqrt{ }$ \\
\hline $\begin{array}{l}\text { Apêndices } \\
\text { vocabulário/ } \\
\text { gramática }\end{array}$ & $\sqrt{ }$ & $\sqrt{ }$ & $\varnothing$ & $\sqrt{ }$ \\
\hline Atividades online & $\varnothing$ & $\sqrt{ }$ & $\varnothing$ & $\varnothing$ \\
\hline $\begin{array}{l}\text { Atividades } \\
\text { fotocopiáveis }\end{array}$ & $\sqrt{ }$ & $\sqrt{ }$ & $\varnothing$ & $\varnothing$ \\
\hline Planos de aula & $\sqrt{ }$ & $\varnothing$ & $\varnothing$ & $\varnothing$ \\
\hline $\begin{array}{l}\text { Outras obras de } \\
\text { referência }\end{array}$ & $\sqrt{ }$ & $\sqrt{ }$ & $\sqrt{ }$ & $\sqrt{ }$ \\
\hline
\end{tabular}

\subsection{Outros recursos}

Além do que já foi descrito nas seções anteriores, os dicionários on-line também podem disponibilizar a função cópia e impressão e oferecer recursos multimídia (som e imagem).

\subsubsection{Cópia e impressão}

O CALD, o MWLD e o WIP permitem que se copiem e imprimam somente as partes selecionadas; o RDEP só copia a página inteira - para imprimir apenas a entrada é necessário copiar a página toda, colar no Word, selecionar a entrada, e então imprimir.

As ilustrações que aparecem MWLD e no WIP também podem ser copiadas e impressas, sendo que o WIP possibilita, ainda a ampliação das imagens. 
QUADRO 9

\section{Cópia e impressão}

\begin{tabular}{|l|c|c|c|c|}
\hline & $\begin{array}{c}\text { CALD } \\
\text { (2009) }\end{array}$ & $\begin{array}{c}\text { MWLD } \\
(2009)\end{array}$ & $\begin{array}{c}\text { RDEP } \\
\text { (2008) }\end{array}$ & $\begin{array}{c}\text { WIP } \\
\text { (2009) }\end{array}$ \\
\hline cópia entradas & $\sqrt{ }$ & $\sqrt{ }$ & $\sqrt{ }$ & $\sqrt{ }$ \\
\hline $\begin{array}{l}\text { cópia } \\
\text { ilustrações }\end{array}$ & - & $\sqrt{ }$ & - & $\sqrt{ }$ \\
\hline $\begin{array}{l}\text { Impressão } \\
\text { entradas }\end{array}$ & $\sqrt{ }$ & $\sqrt{ }$ & $\sqrt{ }$ & $\sqrt{ }$ \\
\hline $\begin{array}{l}\text { Impressão } \\
\text { ilustrações }\end{array}$ & $\sqrt{ }$ & $\sqrt{ }$ & $\sqrt{ }$ & $\sqrt{ }$ \\
\hline
\end{tabular}

\subsubsection{Recursos multimídia}

Dos quatro dicionários analisados, apenas o MWLD e o WIP trazem a pronúncia gravada. O MWLD apresenta o recurso em todas as entradas e o WIP, por depender das contribuições dos usuários, só em alguns casos.

Além das ilustrações, já comentadas na seção 2.3.1, não encontramos nenhuma outra opção multimídia.

\subsection{Síntese das análises}

Organizamos os resultados de nossa análise a partir das duas categorias de aspectos propostas por Hua; Woods (2008): “aspectos relacionados ao conteúdo genérico" [generic-related features] e "aspectos relacionados ao suporte" [media-related features $]^{6}$. Os aspectos genéricos referem-se aos aspectos pertinentes ao conteúdo do dicionário que independem do tipo de suporte, enquanto os aspectos relacionados ao suporte referem-se especificamente ao meio de apresentação - impressa ou eletrônica.

a) Aspectos relacionados ao conteúdo genérico:

- tanto os dicionários monolíngues quanto os bilíngues arrolam os tipos de itens lexicais fundamentais em um dicionário para aprendizes; os únicos itens não contemplados em todos os dicionários são os afixos (que não constituem item essencial nem para a compreensão nem para a produção de textos), os antropônimos e

\footnotetext{
${ }^{6}$ Tradução nossa.
}

topônimos (itens que podem ser buscados em uma enciclopédia);

- os dicionários monolíngues - o CALD e o MWLD - se destacam pela microestrutura detalhada;

- o WIP, por ser um dicionário em construção, tem a microestrutura mais deficiente.

b) Aspectos relacionados ao suporte:

- considerando a estrutura de acesso e os recursos, todos os dicionários on-line pesquisados apresentaram: facilidade de acesso às informações (user-friendly); rapidez na pesquisa; cruzamento de referências; diferentes tipos de busca; possibilidade de impressão do conteúdo e, em alguns casos, de audição da pronúncia;

- os dicionários monolíngues sobressaem por permitirem o acesso a variadas obras de referência de qualidade indiscutível (enriquecendo sobremaneira a pesquisa lexicográfica) e por oferecerem atividades extras (que podem propiciar o desenvolvimento linguístico do aprendiz).

\section{AFUNCIONALIDADEDOSDICIONÁRIOS ONLINE}

Swanepoel (2001, p.167) define a "qualidade funcional" de um dicionário como o grau em que os aspectos da sua concepção (isto é, seu conteúdo, sua estrutura, estilo, layout e meio), dentro dos limites de suas funções e da competência dos seus usuáriosalvo, fornecem a eles, com sucesso, o tipo de suporte lexical que eles precisam para realizar os vários tipos de atividades comunicativas e de aprendizagem nas quais estão engajados em diferentes contextos de uso.

Aplicando essa definição aos dicionários on-line, podemos dizer que a sua "qualidade funcional" estará relacionada não ao seu conteúdo macro e microestrutural (aspectos genéricos), que poderá ser idêntico a um dicionário impresso, mas à sua estrutura de acesso - facilidades de busca e byperlinks - e aos seus recursos midiáticos -imagens e som (aspectos relacionados ao suporte).

Ci. Inf., Brasília, DF, v. 39 n. 3, p.61-72, set./dez., 2010 
Vários estudos apontam vantagens no uso de dicionário on-line.

- Laufer; Hill (2000) utilizaram um dicionário do tipo on-line com explicações em inglês, tradução na língua materna, pronúnica audível e informações extras para estabelecer conexões possíveis entre retenção de vocabulário e comportamento de pesquisa (tipo de informação selecionada e número de consultas para cada item lexical). Os resultados sugeriram que pessoas diferentes têm preferências diferentes, contudo o uso de informações de dicionário com múltiplas informações parece reforçar a retenção.

- As pesquisas de Nesi (2000), Tono (2000) e Loucky (2002) indicam que o uso de dicionários on-line influencia positivamente na compreensão de textos e na aquisição de vocabulário de modo superior ao uso de dicionário impresso em função dos recursos que oferecem e da facilidade dos usuários de ampliar sua pesquisa inicial através dos links para os materiais adicionais.

- Leffa (2006) realizou dois estudos com dicionários on-line - um deles com alunos universitários, falantes de português, lendo textos em língua inglesa e usando dicionários bilíngues convencionais e um dicionário bilíngue eletrônico, e o outro com alunos surdos lendo um texto em português, considerado por eles como língua estrangeira, e usando um dicionário eletrônico bilíngue, LIBRAS-português - e concluiu que esse tipo de dicionário "mais do que o dicionário convencional, tem a potencialidade de antecipar o desempenho de leitores sem a devida competência linguística, levando-os a construir com mais facilidade o sentido do texto" (p.319).

- Hua e Woods (2008) apuraram, a partir de um estudo sobre as preferências e a percepção dos aprendizes em relação aos aspectos genéricos e aos aspectos relacionados ao suporte dos dicionários eletrônicos, que os aspectos considerados úteis pelos usuários são: velocidade de busca, tipo de busca, hiperlinks, tipografia (fonte, cor, tamanho), layout da tela e do texto, som e links externos.
Os resultados de todas essas pesquisas não são conclusivos, entretanto, indicam que as especificidades do suporte on-line são funcionais e, portanto, podem auxiliar nas atividades de produção e compreensão de modo efetivo.

\section{CONSIDERAÇÕES FINAIS}

O objetivo deste artigo foi dar uma visão geral sobre os dicionários do tipo on-line através da revisão dos seus componentes estruturais, da análise comparativa de quatro dicionários disponíveis para o aprendiz de língua inglesa e da apresentação dos resultados de alguns estudos.

Concluímos que o fato de tais ferramentas estarem à disposição tanto dos professores quanto dos aprendizes e de apresentarem tantas facilidades de consulta justifica, ou melhor, exige, que mais estudos empíricos sejam realizados com o intuito de tornálas ainda mais funcionais.

Artigo submetido em 23/09/2010 e aceito em 22/08/2011.

\section{REFERÊNCIAS}

BUGUEÑO, F. Cómo leer y qué esperar de un diccionario monolingue (con especial atención a los diccionarios del español). Revista de Lingua e Literatura, Frederico Westphalen, v. 8/9, p. 97-114, 2003.

; FARIAS, V.S. Informações discretas e discriminantes no artigo léxico. Cadernos de Tradução. v.18, Florianópolis, p. 115-135, 2006.

CARVALHO, O. L. de S. Lexicografia Bilingue Português/Alemão: Teoria e Aplicação à Categoria das Preposições. Brasília: Thesaurus, 2001.

FARIAS, V. S. O exemplo como informação discreta e discriminante em dicionários semasiológicos de língua portuguesa. Alfa: Revista de Linguística, v. 52, n. 1, p. 101-122, 2008.

HALL, C. German dictionaries for the PC.: a survey from the perspective of the language learner. Gf-journal, 2/2000. Disponível em : < http://www.gfl-journal.de/2-2000/hall.html>. Acesso em: 10 maio 2009.

HARTMANN, R.R.K.; JAMES, G. Dictionary of lexicography. London: Routledge, 2001.

HUA, Tan Kim; WOODS, Peter Charles. Media-related or genericrelated features in electronic dictionaries: learners' perception an preferences. GEMA Online Journal of Language Studies, v. 8, n. 2, p.1-17, 2008. 


\section{Isabel Cristina Tedesco Selistre}

JACKSON, H. Lexicography: an introduction. Routledge, 2002.

LAUFER, Batia; HILL, Monica. What lexical information do L2 learners select in a CALL dictionary and how does it affect word retention? Language Learning \& Technology, 3 (2), p.58-76, 2000.

LEECH, G.; NESI, H. Moving towards perfection: the learners' (electronic) dictionary of the future in: HERBST, T.; POPP, K. (eds) The Perfect Learner's Dictionary Lexicographica Series Maior, Tübingen: Max Niemeyer Verlag, 1999, p. 295-308.

LEFFA, Vilson J. O dicionário eletrônico na construção do sentido em língua estrangeira. Cadernos de tradução, Florianópolis, n. 18, p. 319-340, 2006.

LOUCKY, J. P. Improving access to target vocabulary using computerized bilingual dictionaries. ReCALL, v. 14, n. 2, p. $293-$ 312, 2002

NESI, H. Electronic dictionaries in second language vocabulary comprehension and acquisition: the state of the art. In: HEID, U; EVERT, S.; LEHMANN, E.; ROHRER, C. (eds) Proceedings of the Ninth EUR ALEX International Congress, EURALEX, 2000. Stuttgart: Euralex, 2000. v. 2, p. 839-847.

PERUZZO, M. S. Como lidar com os neologismos no texto jornalistico. 2007 Dissertação (Mestrado), Programa de Pós-Graduação em Letras. Porto Alegre: UFRGS, 2007.

POTTIER, B. Gramática del español. Ed. Alcalá S.A. Madrid, 1974.

RIZO-RODRÍGUEZ A. Review of five english learners' dictionaries on cd-rom. Language Learning \& Technology, vol.12, no.1, February 2008, p. 23-42. Disponível em: < http://llt.msu.edu/vol12num1/review1/ default.html $>$.[acesso em: 15 julho 2009]

SCHRYVER, G-M. Lexicographers' dreams in the electronicdictionary age. International Journal of Lexicography, vl.16, no.2, 2003, p.143-199.
SWANEPOEL, P.H. Dictionary quality and dictionary design : a methodology for improving the functional quality of dictionaries. Lexikos 11, 2001, p. 160-190.

TONO, Y. On the effects of different types of electronic dictionary interfaces on L2 learners'reference behaviour in productive/receptive tasks. In U. Heid, S. Evert, E. Lehmann, \& C. Rohrer (Eds.),Proceedings of the ninth Euralex International Congress, EURALEX 2000 (pp. 855-861). Stuttgart:Universität Stuttgart, 2000. Disponível em: <http://www.euralex.org/elx_proceedings/Euralex2000/101_ Yukio $\% 20$ TONO_On $\% 20$ the $\% 20$ Effects $\% 20$ of $\% 20$ Different $\% 20$ Types $\% 20$ of $\% 20$ Electronic $\% 20$ Dictionary $\% 20$ Interfaces $\% 20$ on $\% 20$ L2 $\% 2$ Learners $\% 20$ Reference $\% 20$ Behaviour $\% 20$ in $\% 20$ ProductiveReceptive\%20Tasks.pdf> Acesso em: 28 ago 2010.

WELKER, Herbert Andreas. Dicionários: Uma pequena introdução à lexicografia. Brasília: Thesaurus, 2004.

\section{Dicionários pesquisados:}

CALD $=$ Cambridge Advanced Learner's Dictionary, Cambridge University Press 2009. Disponível em: < http://dictionary.cambridge.org>. [acesso em: 08 junho 2009]

MWLD = Merriam-Webster Learner's Dictionary, Merriam-Webster, Incorporated, 2009 Disponível em: < http:/ /www.learnersdictionary. com>. [acesso em: 08 junho 2009]

RDEP = Reverso Dictionary English/Portuguese HarperCollins Publishers, 2008. Disponível em: http://dictionary.reverso.net/ englishportuguese [acesso em: 08 junho 2009]

WIP = Wiktionary Inglês/Português, 2009 Disponível em: <http:// pt.wiktionary.org/wiki>. [acesso em: 08 junho 2009] 